\title{
Efetividade de intervenções educativas sobre contracepção na adolescência: revisão sistemática da literatura
}

Marcela Lima Silveira Praxedes ${ }^{1}$, Maria Veraci Oliveira Queiroz ${ }^{2}$

\section{RESUMO}

O objetivo foi investigar a efetividade das intervenções educativas sobre contracepção na adolescência. Realizouse busca nas bases de dados da Literatura Latino-Americana e do Caribe (LILACS), Cumulative Index to Nursing \& Allied Health Literature (CINAHL), Web of Science e no portal National Library of Medicine (PubMed/Medline), obedecendo aos critérios de inclusão: estudos que avaliem a efetividade de intervenções educativas sobre contracepção na adolescência, disponíveis na íntegra, nos idiomas português, inglês e espanhol. Observando os critérios de elegibilidade, obteve-se uma amostra de oito artigos. Observaram-se diferentes estratégias educativas sobre contracepção na adolescência utilizadas em diversos contextos e cenários, cujas intervenções mostraramse efetivas, promovendo mudanças no conhecimento e/ou nas atitudes, evidenciando autoeficácia na prevenção de gravidez e infecções sexualmente transmissíveis. Conclui-se, portanto, que as intervenções educativas se mostraram efetivas na promoção da saúde sexual e reprodutiva dos adolescentes.

Descritores: Educação em Saúde; Adolescente; Anticoncepção; Enfermagem Pediátrica.

${ }^{1}$ Enfermeira. Discente do Curso de Mestrado Profissional em Saúde da Criança e do Adolescente da Universidade Estadual do Ceará. Fortaleza, CE, Brasil. E-mail: marcelalsilveira@yahoo.com.br.

${ }^{2}$ Enfermeira, Doutora em Enfermagem. Professora Adjunta da Universidade Estadual do Ceará. Fortaleza, CE, Brasil. E-mail: veracioq@ hotmail.com.

Artigo recebido: 30/01/2018.

Artigo aprovado: 01/10/2018.

Artigo publicado: 31/12/2018.

\section{Como citar esse artigo:}

Praxedes MLS, Queiroz MVO. Efetividade de intervenções educativas sobre contracepção na adolescência: revisão sistemática da literatura. Rev. Eletr. Enf. [Internet]. 2018 [acesso em: ];20:v20a57. Disponível em: https://doi.org/10.5216/ree.v20.51274. 


\section{INTRODUÇÃO}

A adolescência pode ser definida como o período de amadurecimento físico, psicológico e social que marca a passagem da infância para a idade adulta, delimitada cronologicamente pela Organização Mundial da Saúde (OMS) como a faixa etária entre 10 e 19 anos $^{(1)}$.

Os adolescentes possuem peculiaridades próprias de seu desenvolvimento psicoemocional, como a onipotência e desejo de experimentar situações novas e, por isso, tendem a adotar comportamentos de risco, tornando-se mais vulneráveis ${ }^{(2)}$. O despertar da sexualidade surge como um fenômeno psicológico e social, fortemente influenciado pelas crenças pessoais e familiares, e as normas morais da sociedade ${ }^{(3)}$. A iniciação sexual, muitas vezes, ocorre precocemente e associada ao não uso ou uso incorreto dos métodos contraceptivos, com riscos de infecções sexualmente transmissíveis (IST) e uso de álcool e outras drogas, sendo a gravidez não intencional uma das suas grandes consequências, conforme evidenciado na última Pesquisa Nacional sobre a Saúde do Escolar (PeNSE), realizada em 2015(4).

A PeNSE, desenvolvida pelo Instituto Brasileiro de Geografia e Estatística (IBGE) em parceria com o Ministério da Saúde, investigou os fatores de risco e proteção à saúde do adolescente e encontrou que mais de 25\% dos escolares entre 13 e 15 anos de idade e mais de 50\% dos escolares entre 16 e 17 anos de idade já haviam iniciado a vida sexual. Com relação à ocorrência de gravidez e considerando apenas o percentual de meninas que já tiveram relações sexuais, 9\% destas engravidou pelo menos uma vez, com predomínio das meninas de escolas públicas $(9,4 \%)$ e da região Nordeste do país $(13,3 \%)^{(4)}$. A ocorrência de gravidez na adolescência configura-se, portanto, como um problema social e de saúde pública, uma vez que pode ser um risco tanto para o desenvolvimento biopsicossocial da adolescente, como para o desenvolvimento do bebê, com repercussões sociais, econômicas e familiares ${ }^{(5)}$.

Nesse contexto, as práticas educativas em saúde têm se mostrado necessárias, pois estimulam a participação ativa dos sujeitos, fomentando a sua autonomia e protagonismo nas decisões para obtenção de boas ações para a saúde sexual e reprodutiva, podendo ser mediadas por uma grande diversidade de técnicas e materiais ${ }^{(6)}$. Contudo, são fundamentais o acompanhamento e a orientação dos adolescentes, com ampla participação da família, da escola, das instituições de saúde e da comunidade, como meios formadores de opinião(7).

Diversos estudos têm demonstrado a realização de intervenções educativas destinadas à promoção da saúde sexual e reprodutiva do público adolescente ${ }^{(2,8-10)}$. Embora os adolescentes reconheçam a ocorrência de práticas educativas sobre sexualidade, $56 \%$ destes avaliam as intervenções realizadas como insatisfatórias ${ }^{(7)}$. Salienta-se, portanto, a necessidade de investimentos em ações participativas e com recursos tecnológicos que possam estimular o interesse e a participação deste público.

Além disso, é importante guiar-se por Práticas Baseadas em Evidências (PBE), que respondam a uma questão elucidada na prática clínica, dando subsídios para uma conduta segura, com qualidade e baixo custo(11). Nessa perspectiva, realizou-se uma revisão sistemática com o seguinte objetivo: investigar a efetividade das intervenções educativas desenvolvidas sobre a contracepção na adolescência. 


\section{MÉTODOS}

Estudo orientado pela revisão sistemática da literatura, que identifica, seleciona e avalia criticamente estudos relevantes sobre um tema em questão, através de métodos sistemáticos e explícitos, podendo ou não fazer uso de métodos estatísticos (metanálise) para resumir os seus resultados ${ }^{(12)}$. A operacionalização seguiu os sete passos estabelecidos pelo Instituto Cochrane, descritos a seguir: 1) formulação da pergunta, 2) localização e seleção dos estudos, 3) avaliação crítica dos estudos, 4) coleta de dados, 5) análise e apresentação dos dados, 6) interpretação dos dados e 7) aprimoramento e atualização da revisão(13).

Para a formulação da pergunta, seguiu-se a estratégia PICO, onde: Participantes = adolescentes, Intervenção = prática educativa sobre contracepção, Comparação = intervenção padrão ou nenhuma intervenção e Outcomes (desfechos) = aumento do conhecimento sobre contracepção na adolescência. Dessa forma, o estudo foi norteado pela seguinte questão: Qual a efetividade das intervenções educativas sobre a contracepção na adolescência? O acrônimo PICO auxilia na definição correta das evidências necessárias para a pesquisa e evita buscas desnecessárias ${ }^{(14)}$.

A fim de elucidar a questão norteadora, foi realizada uma busca no período de setembro a outubro de 2017 nas bases de dados Literatura Latino-Americana e do Caribe (LILACS), Cumulative Index to Nursing \& Allied Health Literature (CINAHL), Web of Science e National Library of Medicine (PubMed/Medline). Foram utilizados os seguintes descritores controlados entrecruzados com o operador boleano AND: Educação em saúde / Health Education, Contracepção / Contraception e Adolescente / Adolescent, de acordo com a classificação dos Descritores em Ciências da Saúde (DeCS) e do Medical Subject Headings (MeSH/PubMed).

A seleção dos estudos obedeceu aos critérios de inclusão: estudos que avaliem a efetividade/eficácia de intervenções educativas sobre contracepção na adolescência, disponíveis na íntegra, nos idiomas português, inglês e espanhol. Os critérios de exclusão adotados foram: publicações do tipo editorial, cartas ao editor, livros e/ou capítulos de livros, monografias, dissertações, teses, relatos de experiência, estudos de caso e de revisão. Ressalta-se que a busca dos estudos não foi limitada por período de tempo ou ano de publicação dos mesmos, no intuito de abranger o maior número de artigos possíveis. Os artigos encontrados em mais de uma base de dados foram contabilizados apenas uma vez.

A busca inicial resultou em 1.417 artigos. Após a exclusão dos artigos indisponíveis (1.163), realizou-se a seleção por meio da leitura dos títulos e resumos e, quando necessário, dos textos na íntegra, para observar se os mesmos contemplavam a questão norteadora e atendiam aos critérios estabelecidos. Após esta avaliação criteriosa, obteve-se uma amostra final de oito estudos. Foi utilizado o instrumento PRISMA (Preferred Reporting Items for Systematic Review and Meta-Analyses) para explicar a busca e a seleção dos artigos, conforme a Figura $1^{(15)}$.

Para registrar os dados dos artigos, foi utilizado um instrumento de coleta adaptado, a fim de assegurar a relevância dos dados extraídos, assim como minimizar o risco de erros de transcrição e garantir a precisão na checagem das informações ${ }^{(16)}$. E ainda, se classificaram os artigos selecionados de acordo com os níveis de evidência científica do Centro Colaborador do Instituto Joanna Briggs (JBI), a saber: Nível I: revisão sistemática contendo apenas ensaios clínicos controlados randomizados; Nível II: pelo menos um ensaio clínico controlado randomizado; Nível III 1: ensaios clínicos controlados bem delineados, sem randomização; Nível III 2: estudos de 
coorte bem delineados ou caso-controle, estudos analíticos, preferencialmente de mais de um centro ou grupo de pesquisa; Nível III 3: séries temporais múltiplas, com ou sem intervenção e resultados em experimentos não controlados e Nível IV: parecer de autoridades respeitadas, baseadas em critérios clínicos e experiência, estudos descritivos ou relatórios de comitês de especialistas ${ }^{(17)}$.

Figura 1: Fluxograma de seleção dos estudos.

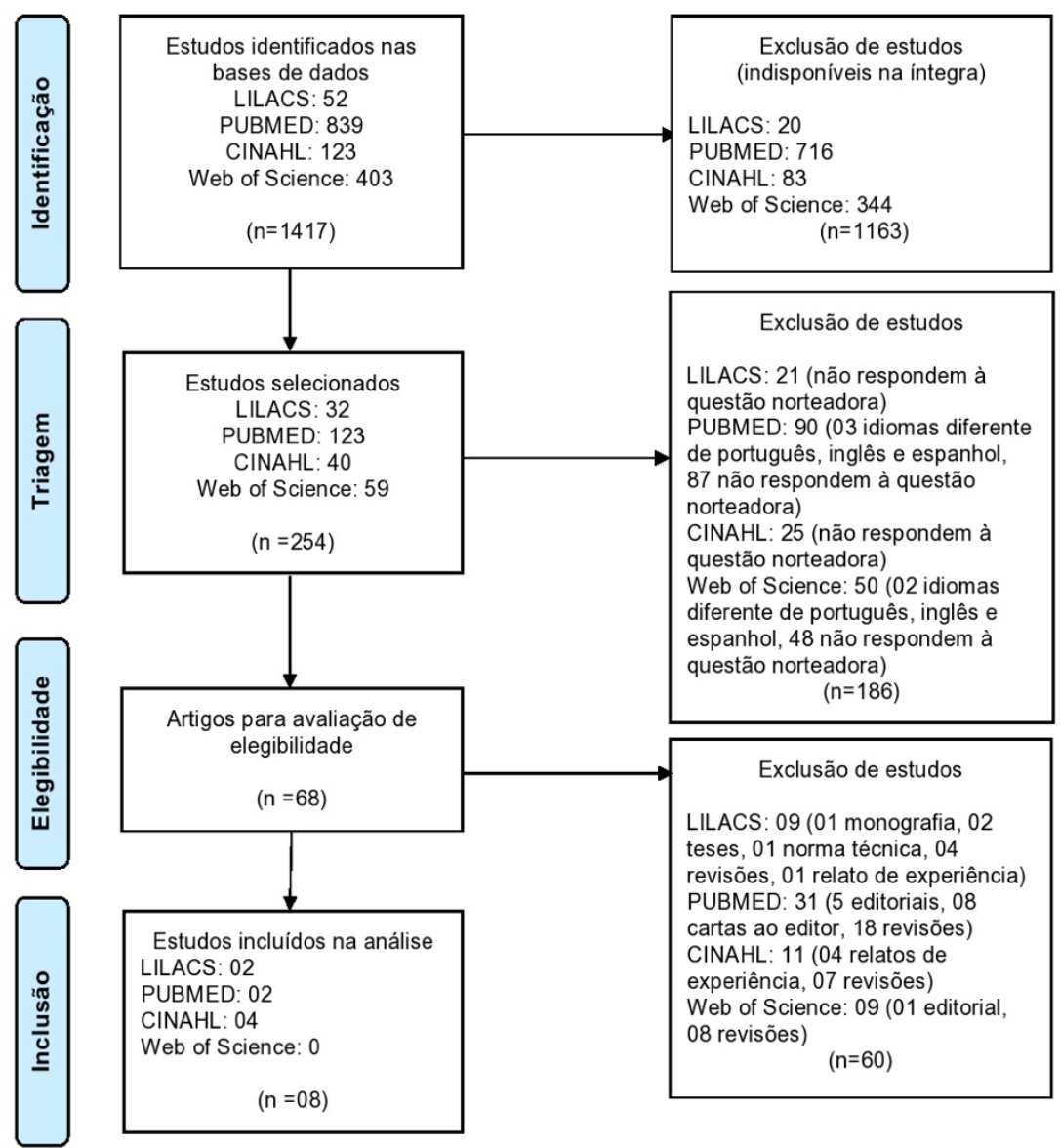

Ademais, para avaliar a qualidade metodológica dos estudos, aplicou-se a escala de qualidade de Jadad ${ }^{(18)}$, que se constitui de três itens relacionados, tendo duas opções de resposta: sim (1 ponto) ou não (zero). Os itens 1 e 2 recebem um ponto adicional caso o método de randomização e mascaramento sejam adequados (Quadro 1). A pontuação da escala varia de zero a cinco, sendo considerados de má qualidade metodológica os estudos com pontuação inferior a dois.

Quadro 1: Escala de qualidade metodológica (Jadad et al., 1996).

\begin{tabular}{|l|}
\hline 1. O estudo foi descrito como aleatório (uso de palavras como "randômico", "aleatório", "randomização")? \\
\hline 1a. O método foi adequado? \\
\hline 2. O estudo foi descrito como duplo-cego? \\
\hline 2a. O método foi adequado? \\
\hline 3. Houve descrição das perdas e exclusões? \\
\hline
\end{tabular}




\section{RESULTADOS}

Dos estudos selecionados sobre a temática que atenderam à questão da pesquisa, obteve-se uma amostra de oito artigos. O Quadro 2 apresenta a síntese dos estudos selecionados, segundo autoria, ano, país, nível de evidência, escala de Jadad(18), objetivos, tipo de estudo, intervenção, amostra e resultados.

Quadro 2: Apresentação da amostra de acordo com autoria, ano, país, nível de evidência, qualidade metodológica, objetivos, tipo de estudo/intervenção, amostra e resultados. Fortaleza, CE, Brasil, 2017.

\begin{tabular}{|c|c|c|c|c|}
\hline $\begin{array}{c}\text { Autoria / Ano / } \\
\text { País / Nível de } \\
\text { evidência / Escala } \\
\text { de Jadad }\end{array}$ & Objetivos & $\begin{array}{l}\text { Tipo de estudo / } \\
\text { Intervenção }\end{array}$ & Amostra & Resultados \\
\hline $\begin{array}{l}\text { Mora et al. / } 2011 \text { / } \\
\text { Cuba / Nível de } \\
\text { Evidência: III } 3 \text { / } \\
\text { Escala de Jadad: } 1\end{array}$ & $\begin{array}{c}\text { Melhorar o } \\
\text { conhecimento dos } \\
\text { adolescentes sobre } \\
\text { aspectos da sexualidade, } \\
\text { com enfoque nos } \\
\text { métodos contraceptivos. }\end{array}$ & $\begin{array}{l}\text { Estudo de intervenção / } \\
\text { Intervenção educativa } \\
\text { através de } 3 \text { encontros } \\
\text { semanais, com o uso de } \\
\text { meios audiovisuais } \\
\text { demonstrativos. }\end{array}$ & $\begin{array}{c}n=62 \\
\text { Idades: } 11-19 \\
\text { anos }\end{array}$ & $\begin{array}{c}\text { A atividade educativa } \\
\text { contribuiu para a melhoria } \\
\text { do conhecimento sobre } \\
\text { contracepção na } \\
\text { adolescência. }\end{array}$ \\
\hline $\begin{array}{l}\text { Hernández et al. / } \\
2010 \text { / Cuba / Nível } \\
\text { de Evidência: III } 3 \text { / } \\
\text { Escala de Jadad: } 2\end{array}$ & $\begin{array}{l}\text { Incrementar o nível de } \\
\text { conhecimento sobre } \\
\text { contracepção nos } \\
\text { adolescentes de uma } \\
\text { escola secundária básica. }\end{array}$ & $\begin{array}{l}\text { Estudo de intervenção } \\
\text { (com randomização) / } \\
\text { Intervenção educativa } \\
\text { através de quatro } \\
\text { módulos que continham } \\
\text { diferentes técnicas } \\
\text { participativas. }\end{array}$ & $\begin{array}{c}\mathrm{n}=136 \\
\text { Idades: não } \\
\text { menciona } \\
\text { (apenas } \\
\text { adolescentes) }\end{array}$ & $\begin{array}{l}\text { A realização da intervenção } \\
\text { mostrou a utilidade do } \\
\text { estudo ao permitir um } \\
\text { aumento do nível de } \\
\text { conhecimento sobre } \\
\text { contracepção em } 57,3 \% \\
\text { dos adolescentes. }\end{array}$ \\
\hline $\begin{array}{l}\text { Sieving et al. / } 2012 \\
\text { / EUA / Nível de } \\
\text { Evidência: II / Escala } \\
\text { de Jadad: } 3\end{array}$ & $\begin{array}{c}\text { Examinar } \\
\text { comportamentos de risco } \\
\text { sexual e mediadores } \\
\text { psicossociais após } 12 \\
\text { meses da intervenção } \\
\text { com o programa Prime } \\
\text { Time. }\end{array}$ & $\begin{array}{l}\text { ECR / Programa Prime } \\
\text { Time, que consiste } \\
\text { numa intervenção } \\
\text { combinada de manejo } \\
\text { individual de casos e } \\
\text { educação liderada pelos } \\
\text { pares. }\end{array}$ & $\begin{array}{l}n=239 \text { (sexo } \\
\text { feminino) } \\
\text { Idades: } 13-17 \\
\quad \text { anos }\end{array}$ & $\begin{array}{l}\text { O grupo intervenção } \\
\text { relatou um uso mais } \\
\text { consistente de } \\
\text { preservativos, } \\
\text { contracepção hormonal e } \\
\text { métodos contraceptivos } \\
\text { combinados (dupla } \\
\text { proteção) do que o grupo } \\
\text { controle. }\end{array}$ \\
\hline $\begin{array}{l}\text { Pérez et al. / } 2005 \text { / } \\
\text { Espanha / Nível de } \\
\text { Evidência: III } 1 \text { / } \\
\text { Escala de Jadad: } 1\end{array}$ & $\begin{array}{l}\text { Avaliar o resultado de } \\
\text { uma intervenção } \\
\text { educativa para prevenir } \\
\text { gravidez indesejada e } \\
\text { infecções sexualmente } \\
\text { transmissíveis (IST) em } \\
\text { adolescentes. }\end{array}$ & $\begin{array}{l}\text { Quase-experimental / A } \\
\text { intervenção se baseou } \\
\text { na organização de um } \\
\text { concurso publicitário } \\
\text { (imprensa, rádio e } \\
\text { televisão) que deviam } \\
\text { ser elaborados pelos } \\
\text { próprios estudantes e } \\
\text { tutelados por um } \\
\text { professor. }\end{array}$ & $\begin{array}{c}\mathrm{n}=197 \\
\text { Idades: } 14-18 \\
\text { anos }\end{array}$ & $\begin{array}{c}\text { Após } 6 \text { meses de } \\
\text { intervenção, houve } \\
\text { melhora significativa no } \\
\text { conhecimento da } \\
\text { capacidade preventiva do } \\
\text { preservativo frente às } \\
\text { IST/AIDS (de } 95,8 \% \text { para } \\
\text { 99,5\%) e no uso correto do } \\
\text { preservativo (de } 62,1 \% \\
\text { para } 73,5 \% \text { ). }\end{array}$ \\
\hline $\begin{array}{c}\text { Gaughran M, } \\
\text { Asgary R. / } 2014 \text { / } \\
\text { Quênia / Nível de } \\
\text { Evidência: III } 3 \text { / } \\
\text { Escala de Jadad: } 1\end{array}$ & $\begin{array}{l}\text { Avaliar a eficácia de um } \\
\text { currículo abrangente } \\
\text { sobre saúde reprodutiva } \\
\text { com foco na prevenção } \\
\text { de IST e gravidez na } \\
\text { adolescência. }\end{array}$ & $\begin{array}{l}\text { Estudo de intervenção / } \\
\text { Implementação de um } \\
\text { currículo de saúde } \\
\text { reprodutiva de } 6 \\
\text { semanas, que incluiu } \\
\text { sessões didáticas, jogos } \\
\text { educacionais e } \\
\text { discussões abertas. }\end{array}$ & $\begin{array}{c}\mathrm{n}=42 \text { (sexo } \\
\text { feminino) } \\
\text { Idades: } 13-19 \\
\text { anos }\end{array}$ & $\begin{array}{l}\text { Observou-se melhora nos } \\
\text { domínios educacionais: } \\
\text { conhecimento sobre } \\
\text { HIV/AIDS (de } 85 \% \text { para } \\
\text { 94\%), conhecimento sobre } \\
\text { gravidez na adolescência e } \\
\text { IST (de 57\% para 82\%) e na } \\
\text { pontuação geral de } \\
\text { conhecimento, atitude e } \\
\text { autoeficácia (de } 81 \% \text { para } \\
\text { 90\%). }\end{array}$ \\
\hline
\end{tabular}




\begin{tabular}{|c|c|c|c|c|}
\hline $\begin{array}{c}\text { Autoria / Ano / } \\
\text { País / Nível de } \\
\text { evidência / Escala } \\
\text { de Jadad }\end{array}$ & Objetivos & $\begin{array}{l}\text { Tipo de estudo / } \\
\text { Intervenção }\end{array}$ & Amostra & Resultados \\
\hline $\begin{array}{l}\text { Zimmerman et al. / } \\
2008 \text { / EUA / Nível } \\
\text { de Evidência: III } 1 \text { / } \\
\text { Escala de Jadad: } 2\end{array}$ & $\begin{array}{l}\text { Avaliar se adaptando um } \\
\text { currículo escolar bem- } \\
\text { sucedido melhora a } \\
\text { eficácia dos adolescentes } \\
\text { na promoção de } \\
\text { comportamentos sexuais } \\
\text { seguros para prevenção } \\
\text { de gravidez e IST. }\end{array}$ & $\begin{array}{l}\text { Quase-experimental / } \\
\text { Duas intervenções: } \\
\text { Currículo escolar de } \\
\text { Redução de Risco } \\
\text { original e modificado. }\end{array}$ & $\begin{array}{c}\mathrm{n}=1.944 \\
\text { Idades: } 13-19 \\
\text { anos }\end{array}$ & $\begin{array}{l}\text { As análises revelam que } \\
\text { não houve diferença } \\
\text { significativa entre os } \\
\text { grupos curriculares em } \\
\text { qualquer uma das variáveis } \\
\text { do estudo. No entanto, os } \\
\text { resultados sugerem que os } \\
\text { estudantes do currículo } \\
\text { modificado foram menos } \\
\text { prováveis de iniciarem a } \\
\text { vida sexual durante a nona } \\
\text { série. }\end{array}$ \\
\hline $\begin{array}{l}\text { Barnet et al. / } 2009 \\
\text { / EUA / Nível de } \\
\text { Evidência: II / Escala } \\
\text { de Jadad: } 3\end{array}$ & $\begin{array}{l}\text { Avaliar a eficácia de uma } \\
\text { intervenção motivacional } \\
\text { assistida por computador } \\
\text { (CAMI) na prevenção de } \\
\text { repetição rápida de } \\
\text { nascimento em mães } \\
\text { adolescentes. }\end{array}$ & $\begin{array}{c}\text { ECR / Intervenção } \\
\text { Motivacional Assistida } \\
\text { por Computador } \\
\text { (CAMI). }\end{array}$ & $\begin{array}{l}\mathrm{n}=235 \text { (sexo } \\
\text { feminino) } \\
\text { Idades: } 12-19 \\
\text { anos. }\end{array}$ & $\begin{array}{c}\text { Receber } 2 \text { ou mais sessões } \\
\text { de CAMI, sozinho ou dentro } \\
\text { de uma intervenção } \\
\text { domiciliar, reduziu o risco } \\
\text { de nascimento reincidente } \\
\text { em mães adolescentes. }\end{array}$ \\
\hline $\begin{array}{l}\text { O'Donnell et al. / } \\
1999 \text { / EUA / Nível } \\
\text { de Evidência: II / } \\
\text { Escala de Jadad: } 3\end{array}$ & $\begin{array}{l}\text { Avaliar a eficácia de um } \\
\text { programa que inclui um } \\
\text { currículo escolar de } \\
\text { alcance para a saúde } \\
\text { associado a um serviço } \\
\text { comunitário para jovens } \\
\text { na redução de } \\
\text { comportamentos sexuais } \\
\text { de risco entre } \\
\text { adolescentes. }\end{array}$ & $\begin{array}{l}\text { ECR / Duas } \\
\text { intervenções: Currículo } \\
\text { escolar modificado de } \\
\text { alcance para a saúde e o } \\
\text { currículo modificado } \\
\text { associado ao serviço } \\
\text { comunitário para } \\
\text { jovens, que } \\
\text { proporcionam aos } \\
\text { estudantes experiências } \\
\text { em serviços que } \\
\text { atendem a comunidade, } \\
\text { explorando suas } \\
\text { habilidades sociais e } \\
\text { comportamentos. }\end{array}$ & $\begin{array}{c}\mathrm{n}=1.061 \\
\text { Idades: } 12 \text { e } 13 \\
\quad \text { anos } \\
\text { (adolescentes do } \\
\text { 70 e 8 ano). }\end{array}$ & $\begin{array}{l}\text { Os resultados mostraram } \\
\text { uma redução de } 15,9 \% \text { na } \\
\text { ocorrência de sexo sem uso } \\
\text { de preservativo e de } 8,4 \% \\
\text { de sexo sem controle de } \\
\text { natalidade (uso de outros } \\
\text { contraceptivos) no grupo } \\
\text { intervenção que recebeu o } \\
\text { currículo modificado } \\
\text { associado ao serviço } \\
\text { comunitário para jovens, } \\
\text { enquanto houve um } \\
\text { discreto aumento em } \\
\text { ambos indicadores no } \\
\text { grupo controle. }\end{array}$ \\
\hline
\end{tabular}

Quanto à caracterização dos artigos, dentre os estudos selecionados, seis (75\%) foram realizados nas Américas, sendo que mais da metade destes foram nos Estados Unidos da América (EUA). Os demais foram desenvolvidos no continente africano e na Europa. Ressalta-se que nenhum estudo realizado no Brasil foi incluído na amostra, por não atenderem aos critérios estabelecidos.

No que se refere ao delineamento metodológico da pesquisa, mais da metade dos estudos apresentaram desenhos de pesquisa com níveis de evidência elevados, consistindo em estudos experimentais (ECR) e quaseexperimentais. Quanto à avaliação da qualidade metodológica, segundo a Escala de Jadad ${ }^{(18)}$, apenas três estudos (37,5\%) foram classificados como de má qualidade metodológica.

O tamanho amostral dos estudos incluídos variou de 42 a 1.944 participantes, perfazendo um total de 3.916 pessoas estudadas. No que concerne às idades dos sujeitos das pesquisas, estas variaram entre 10 e 19 anos, o que caracteriza, cronologicamente, adolescentes, segundo classificação da OMS. Outrossim, três estudos foram realizados apenas com adolescentes do sexo feminino. 


\section{DISCUSSÃO}

A educação em saúde quando possibilita compartilhar conhecimentos na promoção da saúde sexual e reprodutiva dos adolescentes, tende a obter um impacto positivo na mudança de comportamentos de risco, evitando prejuízos para a integridade física, emocional e o convívio social dessa população(7). A maior parte dos estudos incluídos tinham essa finalidade, visando aferir se os resultados da intervenção educativa alcançaram o objetivo com melhorias para a população de adolescentes estudada.

Assim, os resultados mostram que variadas formas de intervenção têm sido utilizadas nas práticas de educação em saúde destinadas ao público adolescente, bem como distintas maneiras de avaliá-las. Dentre os estudos, três realizaram intervenções educativas divididas em sessões grupais, incluindo diferentes técnicas participativas, como palestras, meios audiovisuais, jogos, entre outras ${ }^{(19-21)}$. Outro estudo realizou intervenções por meio de campanhas publicitárias utilizando como recurso: rádio, TV, mídias impressas e distribuição de materiais $^{(22)}$.

É consenso na literatura que as práticas educativas na adolescência devem ser dinâmicas e interativas, através de metodologias participativas, de forma a permitir a comunicação e a expressão dos adolescentes e, por conseguinte, uma maior eficácia no processo ensino-aprendizagem ${ }^{(2,23-24)}$.

Estudos que avaliaram o nível de conhecimento dos adolescentes referente aos métodos contraceptivos já indicavam alguns parâmetros de análise. Um deles ${ }^{(19)}$ mostrou que os métodos mais conhecidos antes da intervenção eram o preservativo masculino, a pílula anticoncepcional oral e o dispositivo intrauterino (DIU) e após a intervenção os adolescentes passaram a conhecer, além destes, o anticoncepcional injetável (100\%) e outros métodos $(27,4 \%)^{(19)}$. A outra pesquisa ${ }^{(20)}$ classificou o conhecimento dos adolescentes em três estágios (bom, regular e mal), revelando um aumento de $47 \%$ na categoria de bom conhecimento, porém não foi especificado quais os tópicos de conhecimento foram abordados no instrumento de avaliação.

Em contrapartida, pesquisadores ${ }^{(21)}$ analisaram além do conhecimento, as atitudes e a autoeficácia dos adolescentes no uso de contraceptivos, evidenciando, após a intervenção de seis semanas, um aumento de $9 \%$ no conhecimento sobre HIV/AIDS e de $25 \%$ sobre as demais IST e gravidez na adolescência, bem como um aumento de $9 \%$ na pontuação geral de conhecimento, atitude e autoeficácia ${ }^{(21)}$. O conceito de autoeficácia vem sendo bastante utilizado nas pesquisas da área da saúde, pois diz respeito a uma crença que pode ser modificada e encorajada nas ações de promoção e educação em saúde, com o intuito de propiciar aos indivíduos, além de conhecimentos, a capacidade e segurança de manter comportamentos e hábitos de vida saudáveis como rotina ${ }^{25-}$ 26).

Um quase-experimento realizado na Espanha analisou o conhecimento de adolescentes escolares sobre o preservativo masculino e o uso correto deste método após um concurso de mensagens publicitárias, por meio da imprensa, rádio e televisão, obtendo um aumento significante de $95,8 \%$ para $99,5 \%$ no conhecimento sobre a capacidade preventiva do preservativo e um aumento de $62,1 \%$ para $73,5 \%$ no uso correto do mesmo ${ }^{(22)}$.

Observa-se que os estudos, mesmo com algumas limitações, apresentam efeito sobre o conhecimento e atitude com possível melhoria na prevenção de IST em adolescentes. A exemplo, estudo que abordou a implantação de programas comunitários inclui a educação por pares no contexto sociocultural em que os adolescentes estão inseridos, revelando um uso mais consistente do preservativo, do anticoncepcional hormonal 
e da dupla proteção no grupo intervenção, sem efeito significativo na diminuição do número de parceiros sexuais ${ }^{(27)}$. Pesquisas que trazem intervenções de educação em saúde sexual e reprodutiva lideradas pelos pares são cada vez mais relatadas na literatura, pois favorecem a troca de conhecimentos e experiências entre pessoas da mesma faixa etária e contexto social, estimulando o protagonismo juvenil e a socialização de saberes ${ }^{(28-29)}$.

Acrescenta-se que dentre os estudos que realizaram intervenções educativas por meio da inclusão de currículos escolares modificados sobre saúde sexual e reprodutiva ${ }^{(30-31)}$, o primeiro mostrou resultados pouco significativos no que diz respeito à mudanças no conhecimento, atitudes, comportamentos e autoeficácia dos adolescentes $^{(30)}$, enquanto no segundo houve diminuição no grupo intervenção de 15,9\% e 8,4\% na ocorrência de sexo sem preservativo e outros métodos contraceptivos, respectivamente, em comparação com um aumento de $3 \%$ e $9 \%$ nos mesmos indicadores do grupo controle ${ }^{(31)}$.

Embora as políticas educacionais brasileiras recomendem a discussão sobre sexualidade de forma transversal nas instituições de ensino, por meio da inclusão do tema nos currículos escolares, são observadas controvérsias entre o ideal e o real, motivada, possivelmente, pela falta de experiência e capacitação dos professores sobre a temática. Nesse sentido, verifica-se a necessidade de ampliação das ações de promoção da saúde sexual e reprodutiva dos adolescentes escolares, por meio de parcerias entre as escolas, universidades e os profissionais de saúde ${ }^{(32-33)}$.

Por fim, o último estudo realizou uma intervenção assistida por computador, agregando uma tecnologia dura ao cuidado educativo. Foi o único estudo realizado com mães adolescentes, com o objetivo de prevenir a recorrência de gravidez nessa população. Os resultados mostram que as adolescentes que participaram da Intervenção Motivacional Assistida por Computador (CAMI) associada à intervenção domiciliar apresentaram um risco significante menor $(13,8 \%)$ de um nascimento subsequente que as que receberam apenas a CAMI (17,2\%) e as que não receberam nenhuma intervenção (grupo controle) $(25 \%)^{(34)}$.

As pesquisas demonstram que o uso de tecnologias na prática educativa contribui para intensificar a adoção de hábitos de vida mais saudáveis, sendo consideradas, ao mesmo tempo, processo e produto. Nesse sentido, é de grande importância a avaliação e validação desses instrumentos ${ }^{(35)}$.

Observa-se que as intervenções foram realizadas nos mais diversos cenários, como escolas(20,22,27,30-31), clínicas comunitárias $^{(19,27)}$, Organizações Não-Governamentais ${ }^{(21)}$ e domicílio(34).

Numa revisão sistemática que analisou a eficácia de intervenções de educação sexual nas escolas, cujos estudos foram publicados entre 2008 e 2010, observou-se melhora significativa a nível de conhecimento, atitudes e comportamentos dos adolescentes em mais de $80 \%$ da amostra, evidenciando a relevância de atuação neste cenário $^{(36)}$.

Quase a totalidade dos estudos analisados $(87,5 \%)$ avaliou a efetividade das intervenções educativas por meio de questionário aplicado em pelo menos dois momentos, antes e após a realização da intervenção. Porém, o tempo de duração da intervenção variou bastante entre os estudos, de quatro semanas a três anos. Percebe-se que as avaliações realizadas a longo prazo permitem, além de investigar o conhecimento adquirido, avaliar de forma mais consistente a mudança de comportamentos dos indivíduos.

Nota-se, portanto, uma heterogeneidade na amostra no que diz respeito ao tipo de intervenção, ao cenário onde foi realizada, ao tempo de duração e aos desfechos analisados. Apesar da dificuldade em comparar os 
achados dos estudos entre si devido a essa heterogeneidade, todos apresentaram algum efeito positivo nos desfechos encontrados, caracterizando a efetividade das intervenções educativas, exceto em uma investigação( ${ }^{(30)}$.

Desse modo, por meio da implementação de estratégias educativas e a consequente avaliação de sua eficácia, é possível mudar paradigmas no processo de educação para a saúde, exigindo cada vez mais criatividade e audácia por parte dos profissionais, de forma a se apropriar de instrumentos inovadores que permitam atingir de forma efetiva a população-alvo, proporcionando reflexão e mudança de prática(37).

\section{CONCLUSÃO}

Com base nos resultados encontrados, pode-se perceber que os pesquisadores da área da saúde têm utilizado variadas estratégias educativas a fim de promover a saúde sexual e reprodutiva dos adolescentes, incluindo diversas metodologias participativas. A efetividade dessas intervenções foi avaliada de distintas formas, essencialmente, aferindo melhoria no conhecimento e/ou mudanças de atitudes e comportamentos de risco, propiciando portanto, possibilidades de prevenção de gravidez precoce e IST. Em sua maioria, os estudos mostraram efeitos positivos das intervenções educativas sobre contracepção na adolescência.

O estudo apresentou como limitação a dificuldade em comparar os resultados dos estudos entre si, devido à diversidade das intervenções, bem como os desfechos analisados para caracterizar a efetividade destas. Além disso, a amostra mostrou-se heterogênea no que diz respeito ao contexto sociocultural em que as intervenções foram implementadas, incluindo estudos desenvolvidos em países das Américas, Europa e África, que apresentam significativas diferenças geográficas, culturais e socioeconômicas, o que pode interferir consideravelmente nos resultados. Contudo, concluiu-se que as intervenções educativas se apresentaram efetivas na melhoria do conhecimento e na competência preventiva com repercussões na saúde sexual e na prevenção da gravidez dos adolescentes.

A inexistência de artigos publicados no Brasil que atendessem aos critérios de seleção da amostra também foi limitadora, não permitindo a análise dos resultados das intervenções que vêm sendo realizadas no cenário nacional e a sua comparação com as outras realidades encontradas. Recomenda-se, portanto, a realização de estudos dessa natureza no país, assim como a incorporação de novas tecnologias às estratégias educativas promotoras de saúde, devido à pertinência de se analisar as intervenções à luz do nosso contexto sociocultural e de saúde. Vale salientar, ainda, a necessidade de pesquisas de avaliação não só das intervenções educativas, mas também dos produtos tecnológicos utilizados, para que os mesmos possam ser incorporados à pratica clínica tendo a garantia de sua efetividade no processo educativo.

\section{REFERÊNCIAS}

1. World Health Organization (WHO). Health for the world's adolescents: a second chance in the second decade [Internet]. 2014 [cited 2017 July 10]. Available from: http://www.who.int/maternal_child_adolescent/documents/second-decade/en/.

2. Ferreira VT, Santos JV, Carvalho AR, Miranda JPL. Vulnerabilidade dos adolescentes do IF Sudeste MG - Campus Juiz de Fora às Doenças Sexualmente Transmissíveis (DSTs). 2017;2(1):42-8.

3. Heilborn ML. Por uma agenda positiva dos direitos sexuais da adolescência. PsicolClin. 2012;24(1):57-68. 
4. Instituto Brasileiro de Geografia e Estatística, Coordenação de População e Indicadores Sociais. Pesquisa nacional de saúde do escolar 2015 [Internet]. Rio de Janeiro: IBGE; 2016 [acesso em: 16 ago. 2018]. 132p. Disponível em:

https://biblioteca.ibge.gov.br/visualizacao/livros/liv97870.pdf.

5. Queiroz MVO, Brasil EGM, Alcântara CM, Carneiro MGO. Perfil da gravidez na adolescência e ocorrências clínico-obstétricas. Rev Rene. 2014;15(3):455-62.

6. Sousa MG, Coelho MMF. Tecnologia educativa sobre sexualidade para promoção da saúde com adolescentes. Rev Diálogos Acadêmicos. 2014;3(2):124-8.

7. Leite CT, Vieira RP, Machado CA, Quirinho GS, Machado MFAS. Prática de educação em saúde percebida por escolares. Cogitare Enferm. 2014;19(1):13-19.

8. Santos CL, Sabóia VM. Sexualidade e saúde na adolescência: relato de experiência. Academus Revista Científica da Saúde. 2017;2(1).

9. Mendes MAR, Pires GPAA, Soares IC, Sampaio CA. Vamos falar sobre gravidez na adolescência? Experiência de educação e saúde em um quilombo. Unimontes Científica. 2017;18(2):158-70.

10. Queiroz MVO, Alcântara CM, Brasil EGM, Silva RM. Participação de adolescentes em ações educativas sobre saúde sexual e contracepção. Rev Bras Promoç Saúde. 2016;29:58-65.

11. Ercole FF, Melo LS, Alcoforado CLGC. Revisão integrativa versus revisão sistemática. [editorial] Rev Min Enferm. 2014;18(1):124.

12. Galvão TF, Pansani TSA, Harrad D. Principais itens para relatar Revisões sistemáticas e Meta-análises: A recomendação PRISMA. Epidemiol Serv Saúde. 2015;24(2):335-42.

13. Higgins JPT, Green S, eds. Cochrane handbook for systematic reviews of interventions. Vol. 4. John Wiley \& Sons, 2011.

14. Galvão TF, Pereira MG. Revisões sistemáticas da literatura: passos para sua elaboração. Epidemiol Serv Saúde. 2014;23(1):183-

4.

15. Fuchs SC, Paim BS. Revisão sistemática de estudos observacionais com metanálise. Rev HCPA. 2010;30(3):294-301.

16. Ursi ES. Prevenção de lesões de pele no perioperatório: revisão integrativa da literatura. [dissertação] Ribeirão Preto: Universidade de São Paulo, 2005.

17. Karino ME, Felli VEA. Enfermagem baseada em evidências: avanços e inovações em revisões sistemáticas. Ciênc Cuid Saúde. 2012;11(5):11-5.

18. Jadad AR, Moore A, Carroll D, Jenkinson C, Reynolds DJM, Gavaghan DJ, et al. Assessing the quality of reports of randomized clinical trials: is blinding necessary?. Controlled clinical trials. 1996;17(1):1-12.

19. Mora MDCG, Gálvez LH, Berrío MN. Intervención educativa sobre algunos aspectos relacionados con la sexualidad en adolescentes de un consultorio del Policlínico “Belkys Sotomayor Álvarez” de Ciego de Ávila. Mediciego. 2011;17(2):2-8.

20. Hernández FA, Guzmán GR, Santelices LPM, Núñez OC. Intervención educativa sobre conocimientos de anticoncepción en adolescentes. Rev AMC. 2010;14(3).

21. Gaughran M, Asgary R. On-site comprehensive curriculum to teach reproductive health to female adolescents in Kenya. Journal of Women's Health. 2014;23(4):358-64.

22. Pérez SC, Martínez BF, Muñoz PM, Martín MTL, Alarcón CF, Castro AV, et al. Intervención educativa para la prevención de embarazos no deseados y enfermedades de transmisión sexual en adolescentes de la ciudad de Toledo. Revista Española de Salud Pública. 2005;79(5):581-9.

23. Mariano MR, Pinheiro AKB, Aquino PS, Ximenes LB, Pagliuca LMF. Jogo educativo na promoção da saúde de adolescentes: revisão integrativa. Rev. Eletr. Enf. [Internet]. 2013 [acesso em: 14 nov. 2017];15(1):265-73. Disponível em:

https://doi.org/10.5216/ree.v15i1.17814.

24. Farias PAM, Martin LAR, Cristo CS. Aprendizagem ativa na educação em saúde: percurso histórico e aplicações. Rev Bras Educ Med. 2015;39(1):143-50.

25. Uchoa JL, Rodrigues AP, Joventino ES, Almeida PCD, Oriá MOB, Ximenes LB. Autoeficácia em amamentar de mulheres no prénatal e no pós-parto: estudo longitudinal. Rev Enferm UFSM. 2016;6(1):10-20.

26. Oliveira RKL, Oliveira BSB, Bezerra JC, Silva MJN, Melo FMS, Joventino ES. Influência de condições socioeconômicas e conhecimentos maternos na autoeficácia para prevenção da diarreia infantil. Esc Anna Nery. 2017;21(4).

27. Sieving RE, McMorris BJ, Beckman KJ, Pettingell SL, Secor-Turner M, Kugler K, et al. Prime Time: 12-month sexual health outcomes of a clinic-based intervention to prevent pregnancy risk behaviors. J Adolesc Health. 2011;49(2):172-9.

28. Santos KB, Murta, SG. Influência dos Pares e Educação por Pares na Prevenção à Violência no Namoro. Psicologia: Ciência e Profissão. 2016;36(4):787-800.

29. Santos MP, Farre AGMC, Bispo MS, Sousa LB, Marinho DDT. Promoção da saúde sexual e reprodutiva de adolescentes: educação por pares. Rev Baiana Enferm. 2017;31(3).

30. Zimmerman RS, Cupp PK, Donohew L, Sionéan CK, Feist-Price S, Helme D. Effects of a School-Based, Theory-Driven HIV and pregnancy prevention curriculum. Perspectives on Sexual and Reproductive Health. 2008;40(1):42-51. 
31. O'donnell L, Stueve A, San Doval A, Duran R, Haber D, Atnafou R, et al. The effectiveness of the Reach for Health Community Youth Service learning program in reducing early and unprotected sex among urban middle school students. American Journal of Public Health. 1999;89(2):176-81.

32. Chaveiro LG, Pires LM, Matos MA, Teles SA, Souza SMB, Souza MM. Análise da temática sexualidade no contexto escolar com professores da educação básica. Rev Rene. 2015;16(5):690-8.

33. Rufino $C B$, Pires $L M$, Oliveira $P C$, Souza $S M B$, Souza $M M$. Educação sexual na prática pedagógica de professores da rede básica de ensino. Rev. Eletr. Enf. [Internet]. 2013 [acesso em: 23 nov. 2017];15(4):983-91. Disponível em: http://dx.doi.org/10.5216/ree.v15i4.19941.

34. Barnet B, Liu J, DeVoe M, Duggan AK, Gold MA, Pecukonis E. Motivational intervention to reduce rapid subsequent births to adolescent mothers: a community-based randomized trial. The Annals of Family Medicine. 2009;7(5):436-45.

35. Dodt RCM, Ximenes LB, Oriá MOB. Validação de álbum seriado para promoção do aleitamento materno. Acta Paul Enferm. 2012;25(2):225-30.

36. Flora Mc, Rodrigues RFF, Paiva HMCGC. Intervenções de educação sexual em adolescentes: uma revisão sistemática da literatura. Rev Enf Ref. 2013;3(10):125-34.

37. Hernández AG, García YR, Sánchez MR. Intervención educativa sobre anticoncepción y adolescência. Rev AMC. 2012;16(1):4552. 